Research, Society and Development, v. 9, n. 10, e8179109213, 2020

(CC BY 4.0) | ISSN 2525-3409 | DOI: http://dx.doi.org/10.33448/rsd-v9i10.9213

\title{
Percepção de usuárias no climatério sobre as práticas integrativas
}

Perception of users in the climacteric about integrative practices Percepción de los usuarios en el climaterio sobre prácticas integradoras

Recebido: 10/10/2020 | Revisado: 18/10/2020 | Aceito: 18/10/2020 | Publicado: 20/10/2020

Thaíse Maria Isnaider Vieira Pilar ORCID: https://orcid.org/0000-0001-7907-5707

Centro Universitário Santo Agostinho, Brasil

E-mail: Thaisnaid4@gmail.com

Vanessa Bonfim Mendes

ORCID: https://orcid.org/0000-0001-6228-3796

Centro Universitário Santo Agostinho, Brasil

E-mail: vbmcristao18@hotmail.com

Nancy Nay Leite Araújo Loiola Batista

ORCID: https://orcid.org/0000-0001-6279-4895

Centro Universitário Santo Agostinho, Brasil

E-mail: nancyloiola@uol.com.br

\section{Resumo}

O objetivo do presente estudo foi identificar em bases cientifica a percepção de mulheres sobre o uso de práticas integrativas como estratégias de alivio das alterações do climatério. Trata-se de uma revisão integrativa de literatura do tipo descritivo, com abordagem qualitativa, os dados coletados através da estratégia PICo e a combinação dos descritores em ciências da saúde (DESCS) A análise das publicações/artigos foi feita através de leituras minuciosas e de forma crítica dos estudos na integra. Foram revisados 12 artigos publicados entre os anos de 2014 e 2020 e que avaliavam a percepção das mulheres a respeito de práticas integrativas. Ao analisar as publicações e artigos encontrados, foram agrupados em Da análise temática emergiram dois temas: Categoria 1. O (des)conhecimento das mulheres em relação ao climatério e o uso de práticas integrativas como forma de tratamento; Categoria 2. A falta de preparo dos enfermeiros e a ausência de estratégias do serviço básico de saúde para lidar com mulheres climatéricas. Evidenciou-se que diversos fatores dificultam a introdução e utilização de terapias complementares como forma de tratamento aos sintomas do climatério, apresentando que se 
necessita de ações públicas que visem proporcionar informações para as usuárias e um melhor preparo do sistema de saúde.

Palavras-chave: Terapias complementares; Atenção básica; Climatério; Mulher; Percepção.

\begin{abstract}
The aim of the present study was to identify, on a scientific basis, the perception of women about the use of integrative practices as strategies to alleviate climacteric changes. It is an integrative literature review of the descriptive type, with a qualitative approach, the data collected through the PICo strategy and the combination of the descriptors in health sciences (DESCS) The analysis of the publications / articles was done through detailed readings and critical form of the studies in their entirety. Twelve articles published between the years 2014 and 2020 were reviewed, evaluating the perception of women regarding integrative practices. Two themes emerged from the thematic analysis: Category 1. The (un) knowledge of women in relation to the climacteric and the use of integrative practices as a form of treatment.; Category 2. The nurses' lack of preparation and the lack of basic health service strategies to deal with climacteric women. It was evidenced that several factors hinder the introduction and use of complementary therapies as a way of treating climacteric symptoms, showing that public actions are needed to provide information to users and a better preparation of the health system.
\end{abstract} Keywords: Complementary therapies; Basic attention; Climacteric; Woman; Perception.

\title{
Resumen
}

El objetivo del presente estudio fue identificar, con base científica, la percepción de las mujeres sobre el uso de prácticas integradoras como estrategias para paliar los cambios climatéricos. Es una revisión integradora de la literatura de tipo descriptivo, con enfoque cualitativo, los datos recolectados a través de la estrategia PICo y la combinación de los descriptores en ciencias de la salud (DESCS) El análisis de las publicaciones / artículos se realizó mediante lecturas detalladas y forma crítica de los estudios en su totalidad. Se revisaron doce artículos publicados entre los años 2014 y 2020, evaluando la percepción de las mujeres sobre las prácticas integradoras. Del análisis temático surgieron dos temas: Categoría 1. El (des) conocimiento de las mujeres en relación al climaterio y el uso de prácticas integradoras como forma de tratamiento; Categoría 2. La falta de preparación de las enfermeras y la falta de estrategias básicas de servicios de salud para atender a las mujeres climatéricas. Se evidenció que varios factores dificultan la introducción y uso de terapias complementarias como forma de 
tratamiento de los síntomas climatéricos, mostrando que se requieren acciones públicas para brindar información a los usuarios y una mejor preparación del sistema de salud.

Palabras clave: Terapias complementarias; Atención básica; Climaterio; Mujer; Percepción.

\section{Introdução}

O climatério é um período biopsicossocial que pode afetar a forma como a mulher vive e responde a essa fase, que representa a passagem da mulher do ciclo reprodutivo para o não reprodutivo, ocorrendo frequentemente entre os 40 e 65 anos (Brasil, 2016).

Esse período climatérico também é acompanhado de sintomas neurovegetativos ou vasomotores como os fogachos, com ou sem sudorese, uma variedade de sintomas neuropsíquicos (alterações de humor, ansiedade e depressão), disfunções sexuais e cefaleia, deste modo, afetando a qualidade de vida. Com isso, podem aparecer de forma casual, na mulher que ainda menstrua regularmente ou, como ocorre com mais frequência, quando iniciam as mudanças do período menstrual (Brasil, 2015).

A Atenção Básica à Saúde propicia o acolhimento e assistência adequada às mulheres no climatério, devido a mesma ser a base para a execução dos cuidados de saúde, prevenção e promoção da saúde de forma que atenda todas as necessidades individuais da população, sendo assim, a principal porta de entrada para o sistema público de saúde (Souza et al., 2017).

As PICS podem ser ofertadas no SUS em todos os ramos da atenção à saúde. Contudo, a PNPIC incentiva que essas práticas sejam implantadas prioritariamente na Atenção Básica. A proposta de PICS é predisposto a toda a Rede de Atenção à Saúde (RAS), sendo ofertada em serviços nos diversos pontos da rede, conforme organização e demanda local. O Ministério da Saúde vem construindo métodos para enfrentar esses desafios a partir da oferta de cursos para os gestores e profissionais de saúde, da publicação das PICS em documentos técnicos do Ministério, como os Cadernos de Atenção Básica com a inserção de indicadores no Programa Nacional de Melhoria do Acesso e da Qualidade na Atenção Básica (PMAQ) e de campos de registro de informação sobre a realização em saúde no e-SUS AB e ações de socialização (Brasil, 2018).

Diante disso, as práticas integrativas tem sido proposta de uma nova abordagem, destacando a importância de uma escuta qualificada e uma visão holística à mulher no climatério correlacionando com a intervenção clinica necessária, ou seja, tratando os sintomas desagradáveis e contemplando a integralidade, permitindo uma melhor percepção da mulher nesse período climatérico, com a atuação da assistência acolhedora do enfermeiro, retirando as 
principais dúvidas sobre o climatério e incentivando para a prática integrativa mais adequada.

Espera-se contribuir com este estudo como subsidio para outras pesquisas de modo que seja constituído um conhecimento acadêmico embasado na ciência no uso das práticas integrativas, visando identificar em bases cientificas a percepção de mulheres sobre o uso de práticas integrativas como estratégias de alívio às alterações do climatério, já que o conhecimento dessas práticas ainda é incipiente e inacessível a uma boa parcela da comunidade.

A pesquisa poderá servir como um estímulo aos profissionais de saúde, especialmente os enfermeiros, para que busquem conhecimento nessas práticas complementares e assim, inclui-las em sua assistência às mulheres no climatério, auxiliando a correlacionar e executar cada tipo de prática integrativa com alguma alteração específica do climatério, de maneira que esse período seja vivido de forma agradável e com uma melhor qualidade de vida.

\section{Metodologia}

Trata-se de uma revisão integrativa de literatura, do tipo descritivo, com abordagem qualitativa, que busca responder a seguinte questão norteadora: "Qual a percepção de mulheres no climatério sobre o uso de práticas integrativas, como estratégias de alivio de alterações próprias do climatério?”.

Revisão integrativa é um tipo de pesquisa que fornece informações mais amplas de maneira sistemática, ordenada e abrangente, sobre um assunto ou tema, com finalidade de sintetizar resultados obtidos em pesquisa sobre temas ou questões. A definição das informações a serem extraídas dos estudos selecionados por categorização; avaliação dos estudos incluídos; interpretação dos resultados; e apresentação da revisão/síntese do conhecimento (Ercole et al., 2015).

Após a definição do tema do estudo, realizou-se um levantamento bibliográfico na base de dados da Biblioteca Virtual em Saúde (BVS), nos meses de Março a Setembro de 2020, utilizando artigos indexados nas bases de dados Scientif Eletronic Librany Online (SCIELO), Literatura Latino-Americano e do Caribe em Ciências da Saúde (LILACS) e Medical Literature Analysisand Retrieval System Online (MEDLINE), BDENF-ENFERMAGEM, utilizando a estratégia PICo, onde se teve acesso aos artigos na integra.

Para o estudo, foi utilizado a combinação dos descritores: "Mulher"; "Climatério"; "Percepção"; "Terapias Complementares"; “Atenção Básica"; conforme apresentação do vocabulário contido nos Descritores em Ciências da Saúde (Decs), validados pela BVS e cruzadas entre si por meio do operador booleano AND. 
Research, Society and Development, v. 9, n. 10, e8179109213, 2020

(CC BY 4.0) | ISSN 2525-3409 | DOI: http://dx.doi.org/10.33448/rsd-v9i10.9213

Definiu-se como critérios de inclusão publicações no período de 2014 a 2020, artigos completos disponíveis em língua portuguesa e inglesa. Os critérios de exclusão foram artigos fora do período de inclusão de 2014 a 2020, artigos de línguas distintas dos artigos utilizados, exceto, publicações que não respondiam à questão norteadora e objetivo do estudo.

A partir da aplicação da estratégia PICo e dos descritores utilizados foram encontradas 1018 publicações, após a aplicação dos critérios de inclusão e exclusão totalizou-se uma amostra parcial 222 artigos, depois de uma leitura mais precisa aderiu-se um total final de 12 publicações de acordo para serem trabalhados no estudo.

Figura 1. Fluxograma de seleção dos artigos para a elaboração da revisão integrativa.

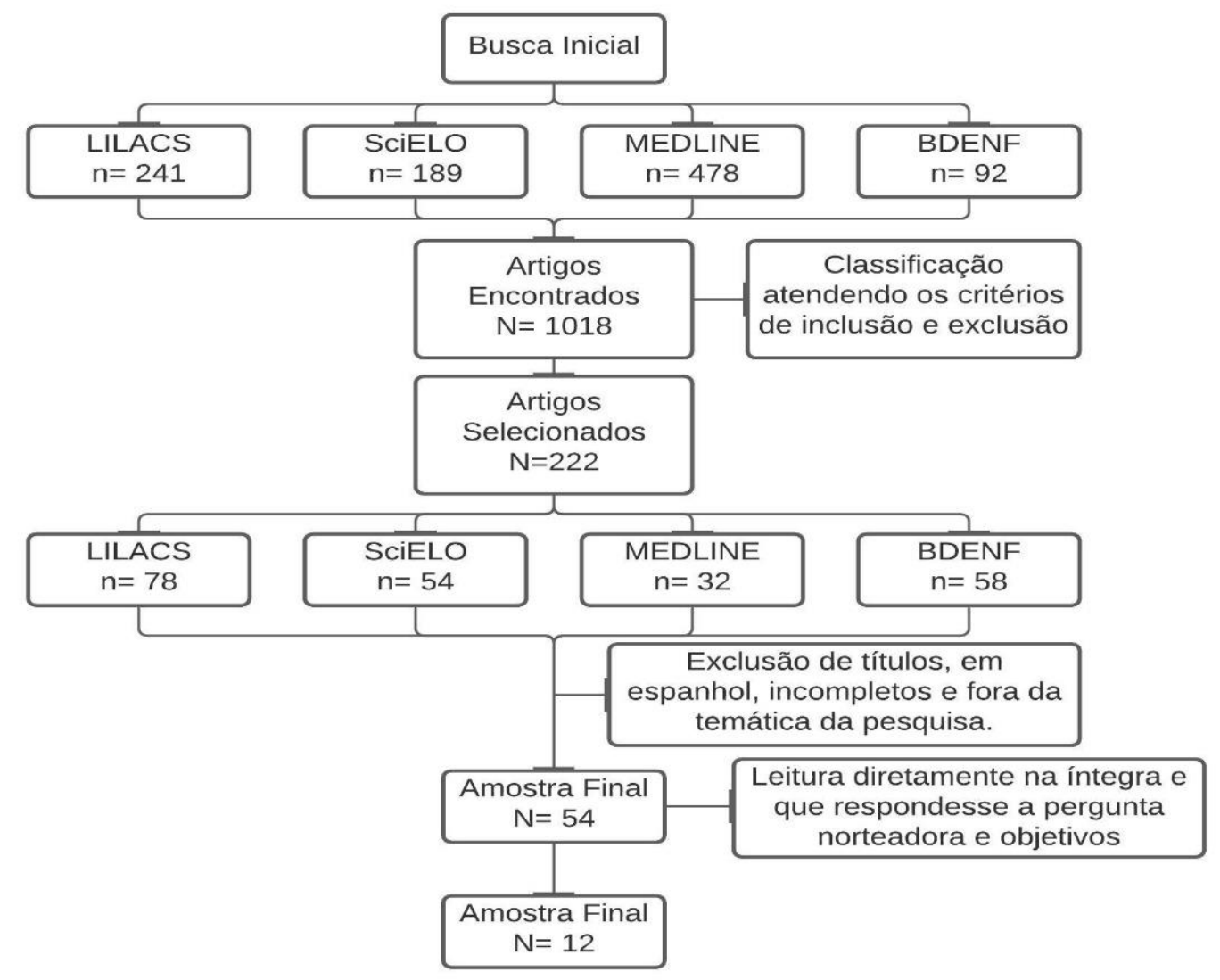

Fonte: Autores.

O fluxograma acima apresenta as etapas do processo de pesquisa e seleção das obras escolhidas para esta revisão, demonstrando a ordem de filtro e resultados apresentados.

A análise das publicações/artigos foi feita através de leituras minuciosas e de forma crítica dos estudos na integra, sendo extraídas as principais ideias de cada autor considerando principalmente os resultados evidenciados e as conclusões de cada publicação componente na 
amostra.

A construção desse artigo foi pautada nas normas legais dispostas pela Associação Brasileira de Normas Técnicas (ABNT) e foi reproduzida fielmente a ideia dos autores.

\section{Resultados e Discussão}

Com base na literatura obtivemos como amostra final 12 artigos selecionados de acordo com os critérios de inclusão e exclusão.

Todos os artigos se encontram disponíveis gratuitamente na Biblioteca virtual de Saúde (BVS), estes artigos estão caracterizados no Quadro 1 abaixo contendo as seguintes variáveis: título, metodologia, autor, ano e periódico.

Quadro 1. Caracterização da amostra dos artigos, Teresina-PI, 2020.

\begin{tabular}{|c|c|c|c|}
\hline $\mathbf{N}^{\mathbf{o}}$ & TÍTULO & $\begin{array}{c}\text { METODOLOGIA, AUTOR } \\
\text { E ANO }\end{array}$ & PERIODICO \\
\hline A1 & $\begin{array}{l}\text { Atuação de enfermeiros na atenção } \\
\text { às mulheres no climatério. }\end{array}$ & $\begin{array}{l}\text { Estudo do tipo descritivo, com } \\
\text { abordagem qualitativa. Silva } \\
\text { et al., (2015). }\end{array}$ & $\begin{array}{l}\text { Revista de } \\
\text { Enfermagem } \\
\text { UFPE Online. }\end{array}$ \\
\hline A2 & $\begin{array}{l}\text { Vivenciando o climatério: } \\
\text { percepções e vivências de mulheres } \\
\text { atendidas na atenção básica. }\end{array}$ & $\begin{array}{l}\text { Estudo do tipo descritivo, com } \\
\text { abordagem qualitativa. Vieira } \\
\text { et al., (2018). }\end{array}$ & $\begin{array}{l}\text { Revista } \\
\text { Enfermagem em } \\
\text { Foco. }\end{array}$ \\
\hline A3 & $\begin{array}{l}\text { Cuidado de enfermagem no } \\
\text { climatério: } \\
\text { desmedicalizadora na atenção } \\
\text { primária de saúde. }\end{array}$ & $\begin{array}{l}\text { Revisão integrativa. Oliveira, } \\
\text { et al., (2017). }\end{array}$ & $\begin{array}{l}\text { Revista de } \\
\text { Enfermagem } \\
\text { UFPE Online. }\end{array}$ \\
\hline A4 & $\begin{array}{l}\text { Potenciais interações relacionadas } \\
\text { ao uso de medicamentos, plantas } \\
\text { medicinais e fitoterápicos em } \\
\text { mulheres no período do climatério. }\end{array}$ & $\begin{array}{l}\text { Estudo do tipo descritivo e } \\
\text { analítico. Gellati et al., (2016). }\end{array}$ & $\begin{array}{l}\text { Revista de } \\
\text { Pesquisa Cuidado } \\
\text { é Fundamental } \\
\text { Online. }\end{array}$ \\
\hline A5 & $\begin{array}{l}\text { Fatores associados à autopercepção } \\
\text { negativa de saúde em mulheres } \\
\text { climatéricas. }\end{array}$ & $\begin{array}{l}\text { Estudo transversal. } \\
\text { Silva, et al., (2018). }\end{array}$ & $\begin{array}{l}\text { Revista Ciência \& } \\
\text { Saúde Coletiva. }\end{array}$ \\
\hline A6 & Percepções e sentimentos sobre as & Estudo do tipo qualitativo com & Revista Gaúcha de \\
\hline
\end{tabular}


Research, Society and Development, v. 9, n. 10, e8179109213, 2020

(CC BY 4.0) | ISSN 2525-3409 | DOI: http://dx.doi.org/10.33448/rsd-v9i10.9213

\begin{tabular}{|c|c|c|c|}
\hline & $\begin{array}{l}\text { alterações corporais de mulheres } \\
\text { climatéricas. }\end{array}$ & $\begin{array}{l}\text { caráter exploratório- } \\
\text { descritivo. Curta et al., (2020). }\end{array}$ & Enfermagem. \\
\hline A7 & Terapias alternativas no climatério. & $\begin{array}{l}\text { Revisão da literatura. Barra et } \\
\text { al., (2014). }\end{array}$ & Revista FEMINA. \\
\hline A8 & $\begin{array}{l}\text { Terapias complementares na } \\
\text { redução de sintomas do climatério: } \\
\text { Ensaio clínico. }\end{array}$ & $\begin{array}{l}\text { Estudo clínico randomizado. } \\
\text { Leão et al., (2015). }\end{array}$ & $\begin{array}{l}\text { Cadernos de } \\
\text { Naturologia e } \\
\text { Terapias } \\
\text { Complementares. }\end{array}$ \\
\hline A9 & $\begin{array}{l}\text { Terapias complementares: } \\
\text { fitoterapia como opção terapêutica } \\
\text { no climatério e menopausa. }\end{array}$ & $\begin{array}{l}\text { Revisão de literatura. Rocha, } \\
\text { et al., (2018). }\end{array}$ & $\begin{array}{l}\text { Revista de ciências } \\
\text { da saúde nova } \\
\text { esperança. }\end{array}$ \\
\hline A10 & $\begin{array}{l}\text { A utilização de fitoterápicos no } \\
\text { manejo de mulheres no } \\
\text { climatério/menopausa. }\end{array}$ & $\begin{array}{l}\text { Revisão integrativa, com } \\
\text { abordagem quantitativa. } \\
\text { Souza et al., (2020). }\end{array}$ & $\begin{array}{l}\text { Research, Society } \\
\text { and Development. }\end{array}$ \\
\hline A11 & $\begin{array}{l}\text { Acupuntura no tratamento da } \\
\text { ansiedade no climatério: } \\
\text { terapêutica complementar na } \\
\text { promoção da saúde mental. }\end{array}$ & $\begin{array}{l}\text { Estudo experimental, misto } \\
\text { (uni-cego e aberto), } \\
\text { randomizado. Girão et al., } \\
\text { (2014). }\end{array}$ & $\begin{array}{l}\text { Revista } \\
\text { enfermagem } \\
\text { UFPE online. }\end{array}$ \\
\hline A12 & $\begin{array}{l}\text { As alterações biopsicossociais no } \\
\text { climatério e a inter-relação com a } \\
\text { qualidade de vida: um estudo de } \\
\text { revisão integrativa. }\end{array}$ & $\begin{array}{l}\text { Revisão integrativa. Moreira, } \\
\text { et al., (2014). }\end{array}$ & $\begin{array}{l}\text { Revista } \\
\text { Memorialidades. }\end{array}$ \\
\hline
\end{tabular}

Fonte: BVS - Biblioteca Virtual de Saúde.

O quadro acima apresenta os artigos utilizados para o estudo e produção desta revisão integrativa. Os itens foram identificados como A1 à A12, apresentando respectivamente, título da obra, metodologia utilizada, autores, ano de publicação e a revista à qual foi publicada. 
Research, Society and Development, v. 9, n. 10, e8179109213, 2020

(CC BY 4.0) | ISSN 2525-3409 | DOI: http://dx.doi.org/10.33448/rsd-v9i10.9213

Gráfico 1. Distribuição dos artigos publicados pela variável: ano, entre os anos de 2014 a 2020. Teresina-PI, 2020.

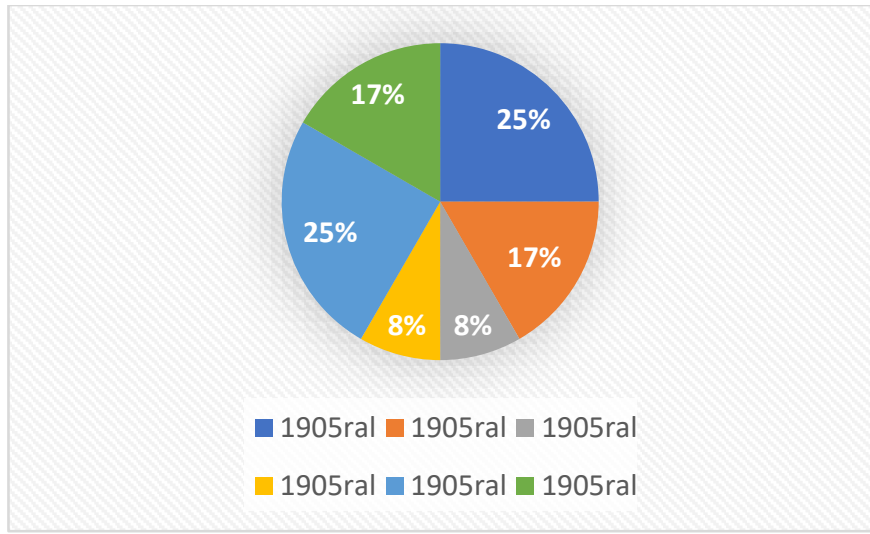

Fonte: BVS - Biblioteca Virtual de Saúde.

O Gráfico 1 acima mostra a análise dos artigos utilizando a variável ano, podemos observar que foram de 2014 e 2018 (25\%), 2016 e 2017 (8\%), 2015 e 2020 (17\%).

Contudo após a caracterização dos artigos selecionados, foi possível analisar os resultados em evidência de todos os artigos demostrados logo abaixo no Quadro 2, levando em relação os números de estudos, título de acordo com idioma que foi publicado, e resultados em evidências.

Quadro 2. Resultados em evidência dos artigos selecionados. Teresina - PI, 2020.

\begin{tabular}{|c|c|c|}
\hline $\mathbf{N}^{\mathbf{o}}$ & TÍTULO & RESULTADO EM EVIDÊNCIAS \\
\hline A1 & $\begin{array}{l}\text { Atuação de } \\
\text { enfermeiros na } \\
\text { atenção às mulheres } \\
\text { no climatério. }\end{array}$ & $\begin{array}{l}\text { Evidenciou-se que o climatério não é tratado como devido, e } \\
\text { sem a merecida importância, muitas vezes não sendo prioridade } \\
\text { de atendimento para essas mulheres. A assistência é } \\
\text { caracterizada por ações dispersas associadas a outras atividades, } \\
\text { como por exemplo, no grupo de hipertensos, confirmando a } \\
\text { inexistência de estratégias específicas para mulheres no período } \\
\text { do climatério nas UBS pesquisadas. }\end{array}$ \\
\hline A2 & $\begin{array}{l}\text { Vivenciando o } \\
\text { climatério: percepções } \\
\text { e vivências de } \\
\text { mulheres atendidas na } \\
\text { atenção básica. }\end{array}$ & $\begin{array}{l}\text { O estudo apresentou que as entrevistadas restringem o } \\
\text { climatério à ocorrência da menopausa. Portanto, reitera-se a } \\
\text { necessidade dos profissionais da APS em acolher e atender essa } \\
\text { demanda de modo a proporcionar a mulher conhecimento com } \\
\text { relação ao climatério e melhora dos sintomas, uma vez que } \\
\text { influenciam diretamente na qualidade de vida. }\end{array}$ \\
\hline
\end{tabular}




\begin{tabular}{|c|c|c|}
\hline A3 & $\begin{array}{l}\text { Cuidado de } \\
\text { enfermagem no } \\
\text { climatério: perspectiva } \\
\text { desmedicalizadora na } \\
\text { atenção primária de } \\
\text { saúde. }\end{array}$ & $\begin{array}{l}\text { Evidencia-se a necessidade de uma atenção à mulher que } \\
\text { vivencia o climatério, considerando esta como um processo } \\
\text { fisiológico, parte de seu ciclo vital. Reforça também para uma } \\
\text { urgência na reformulação dos serviços de saúde na atenção } \\
\text { primária, na perspectiva do autocuidado, no sentido de } \\
\text { colaborar para que as mulheres criem mecanismos de autonomia } \\
\text { nessa fase da vida, a partir de uma perspectiva } \\
\text { desmedicalizadora. }\end{array}$ \\
\hline $\mathbf{A}$ & $\begin{array}{l}\text { Potenciais interações } \\
\text { relacionadas ao uso de } \\
\text { medicamentos, plantas } \\
\text { medicinais } \\
\text { fitoterápicos em } \\
\text { mulheres no período } \\
\text { do climatério. }\end{array}$ & $\begin{array}{l}\text { Evidenciaram que a popularidade do uso de produtos naturais } \\
\text { torna importante o entendimento dos potenciais interações entre } \\
\text { plantas medicinais, fitoterápicos e medicamentos, bem como o } \\
\text { acompanhamento do uso concomitante, pelos profissionais de } \\
\text { saúde, visando identificar a ocorrência de interações que podem } \\
\text { comprometer a saúde do usuário. }\end{array}$ \\
\hline A5 & $\begin{array}{l}\text { atores associados à } \\
\text { utopercepção } \\
\text { egativa de saúde em } \\
\text { uulheres climatéricas. }\end{array}$ & $\begin{array}{l}\text { O presente estudo revelou uma elevada prevalência de } \\
\text { autopercepção negativa da saúde (saúde regular ou ruim) entre } \\
\text { as mulheres climatéricas. }\end{array}$ \\
\hline A6 & $\begin{array}{l}\text { Percepções } \\
\text { sentimentos sobre as } \\
\text { alterações corporais de } \\
\text { mulheres climatéricas. }\end{array}$ & $\begin{array}{l}\text { Foi possível identificar que elas possuem poucas informações } \\
\text { ou conhecimentos sobre o climatério, identificado, por elas, } \\
\text { como a pré ou pós-menopausa, ou a própria menopausa em si, e } \\
\text { foi possível saber o que sentem relatar as queixas ou benefícios } \\
\text { que vivenciaram com o final das menstruações. Percebe-se, } \\
\text { também, que a sua individualidade, ou seja, a sua personalidade, } \\
\text { contribui para como cada mulher vive esta fase, seja de um jeito } \\
\text { positivo ou negativo. }\end{array}$ \\
\hline A7 & $\begin{array}{l}\text { Terapias alternativas } \\
\text { no climatério. }\end{array}$ & $\begin{array}{l}\text { Ao analisar através de bases cientificas, evidencia os benefícios } \\
\text { das Terapias alternativas á TH na redução dos sintomas } \\
\text { Climatéricos, como estratégia de alivio. }\end{array}$ \\
\hline A8 & $\begin{array}{l}\text { Terapias } \\
\text { complementares na }\end{array}$ & $\begin{array}{l}\text { O estudo mostra uma análise na eficácia da Terapia floral, } \\
\text { Auriculoterapia, Toque terapêutico no controle de sintomas } \\
\text { climatéricos, correlacionando com alguma sintomatologia para }\end{array}$ \\
\hline
\end{tabular}


Research, Society and Development, v. 9, n. 10, e8179109213, 2020

(CC BY 4.0) | ISSN 2525-3409 | DOI: http://dx.doi.org/10.33448/rsd-v9i10.9213

\begin{tabular}{|c|c|c|}
\hline & $\begin{array}{l}\text { do climatério: Ensaio } \\
\text { clínico. }\end{array}$ & $\begin{array}{l}\text { o alivio dos sintomas, onde evidencia uma redução nos sintomas } \\
\text { no climatério, através das terapias complementares. }\end{array}$ \\
\hline A9 & $\begin{array}{l}\text { Terapias } \\
\text { complementares: } \\
\text { fitoterapia como opção } \\
\text { terapêutica no } \\
\text { climatério } \\
\text { menopausa. }\end{array}$ & $\begin{array}{l}\text { O estudo evidencia que a busca do alivio dos sintomas e a partir } \\
\text { dos conhecimentos dos efeitos colaterais da terapia de } \\
\text { reposição hormonal muitas mulheres recorrem a terapia } \\
\text { alternativa usando como tratamento, os fitoterápicos como } \\
\text { recurso alternativa para o tratamento e alivio dos sintomas no } \\
\text { climatério. }\end{array}$ \\
\hline A10 & $\begin{array}{l}\text { A utilização de } \\
\text { fitoterápicos no manejo } \\
\text { de mulheres no } \\
\text { climatério/menopausa. }\end{array}$ & $\begin{array}{l}\text { O estudo mostrou que os fitoterápicos abordados apresentam } \\
\text { resultados significativos para as queixas climatéricas } \\
\text { manifestadas em mulheres saudáveis, sendo uma boa } \\
\text { alternativa terapêutica para o seu tratamento especifico. }\end{array}$ \\
\hline A11 & $\begin{array}{lr}\text { Acupuntura } & \text { no } \\
\text { tratamento } & \text { da } \\
\text { ansiedade } & \text { no } \\
\text { climatério: terapêutica } \\
\text { complementar na } \\
\text { promoção da saúde } \\
\text { mental. }\end{array}$ & $\begin{array}{l}\text { Ao analisar o efeito da acupuntura na ansiedade em mulheres } \\
\text { no climatério e nos sintomas psicofísicos relacionados a } \\
\text { síndrome climatérica, evidenciou-se a redução da ansiedade e } \\
\text { dos sintomas relacionados a esse período do climatério, com a } \\
\text { pratica da acupuntura nas mulheres que vivenciam esse } \\
\text { período. }\end{array}$ \\
\hline A12 & $\begin{array}{l}\text { As alterações } \\
\text { biopsicossociais no } \\
\text { climatério e a inter- } \\
\text { relação com a } \\
\text { qualidade de } \\
\text { estuda } \\
\text { integrativa. }\end{array}$ & $\begin{array}{l}\text { Evidenciou-se que as principais alterações no climatério foram } \\
\text { depleção hormonal, sintomas vasomotores e alterações } \\
\text { geniturinárias, irritabilidade. Contundo a uma procura muito } \\
\text { evidente no aumento de expectativa de vida e maior } \\
\text { longevidade feminina, trazendo a necessidade da procura da } \\
\text { mulher a Atenção Básica a Saúde. }\end{array}$ \\
\hline
\end{tabular}

Fonte: BVS - Biblioteca Virtual de Saúde.

O quadro acima apresenta os artigos utilizados para o estudo e produção desta revisão integrativa. Os itens foram identificados como A1 à A12, apresentando um resumo de seus resultados, visando buscar uma melhor organização em temas para que possa haver uma melhor separação de categorias para a discussão a seguir.

Ao analisar os artigos e as evidências encontradas, os artigos foram correlacionados em 
duas categorias:

\subsection{O (des)conhecimento das mulheres em relação ao climatério e o uso de práticas integrativas como forma de tratamento.}

Os artigos A2, A5, A6 e A12 são pesquisas em que as mulheres participantes referem seu conhecimento sobre o climatério e seus sintomas.

De acordo com Vieira et al., (2018) e Curta et al., (2020) concordam que a as mulheres possuem um conhecimento adequado sobre o climatério e seus sintomas, relatam insônia, alterações de humor, cansaço, dor de cabeça, palpitação, redução da libido, diminuição da lubrificação vaginal, deposição de gordura no abdome e flancos, mas desconhecem formas de tratamento disponível para combater os sintomas no geral além de fármacos.

Para Silva et al., (2018) e Moreira et al., (2014) os estudos apresentaram uma alta prevalência de autopercepção negativa da saúde (saúde regular ou ruim) entre as mulheres climatéricas, devido por terem o conhecimento a respeito dos sintomas, mas não buscarem formas de tratamento ou não receberem estímulos e informações a respeito das formas de tratamento pelas unidades responsáveis de saúde de suas regiões, como práticas integrativas.

Os artigos A7, A8, A9, A10 e A11 são pesquisas em que se referem ao uso de práticas integrativas como formas de tratamento para sintomas do climatério e sobre a efetividade dessa forma de tratamento.

Barra et al., (2014) e Leão et al., (2015) evidenciam os benefícios das terapias alternativas no tratamento de sintomas climatéricos como estratégia de alívio, servindo como substituição à Terapia Hormonal $(\mathrm{TH})$. A pesquisa demonstra a eficácia de terapias, como a terapia floral, auriculoterapia e toque terapêutico no controle dos sintomas climatéricos.

Rocha et al., (2018) e Souza et al., (2020) concordam que a utilização da fitoterapia como opção terapêutica no climatério apresenta resultados significativos para as queixas comumente apresentadas pelas climatéricas, a pesquisa também apresenta um grande aumento pela busca sobre fitoterapia. Isso se deve aos diversos efeitos colaterais da terapia de reposição hormonal que muitas mulheres recorrem normalmente, apresentando a fitoterapia como uma ótima terapia substituta.

Já Girão et al. (2014) apresenta a acupuntura como uma forte arma no combate aos sintomas climatéricos, como a ansiedade. Como resultado de análise do estudo, evidenciou-se que uma redução significativa dos sintomas psicofísicos relacionados à síndrome climatérica, servindo como terapia complementar para uma melhora de saúde mental tão importante no 
período climatérico.

\subsection{A falta de preparo dos enfermeiros e a ausência de estratégias do serviço básico de saúde para lidar com mulheres climatéricas}

Os artigos A1, A3 e A4 são pesquisas que apresentam os atuais problemas no sistema básico de saúde, reforçando a falta de preparo dos enfermeiros, a quase nula disseminação de informação para as mulheres climatéricas e a ausência de estímulos para o autocuidado da mulher.

Silva et al., (2015) apresenta que o climatério não é tratado da forma que deveria e sem a merecida importância, muitas vezes deixando de ser a prioridade de atendimento nos locais de saúde, como associar os sintomas climatéricos da mulher à outras atividades como hipertensão, reafirmando e demonstrando a ausência de estratégias para lidarem com essas mulheres.

Oliveira et al., (2017) e Gellati et al., (2016) concordam que a atenção primária de saúde deveria estabelecer uma visão desmedicalizadora, reforçando para uma urgente reformulação dos serviços de saúde, no sentido de perspectiva do autocuidado, visando fazer as mulheres criarem formas de autonomia de cuidado, como o fornecimento de informações a respeito da fitoterapia, orientando de forma que uso de plantas medicinais e produtos naturais possam vir a combater os sintomas do climatério e assim evitar aglomerações em unidades básicas de saúde e servindo como educação de saúde para mulheres nesse estágio de sua vida.

\section{Considerações Finais}

A realização do presente estudo possibilitou analisar a percepção de mulheres no climatério sobre as práticas integrativas.

Todos os objetivos deste estudo foram alcançados e, a partir dos dados analisados, podese perceber que na literatura existem poucas publicações, principalmente recentes, que envolvam o uso de práticas integrativas em mulheres no período climatérico.

Nas amostras estudadas, pode-se perceber que existem lacunas ou até mesmo o desconhecimento total das mulheres sobre o climatério, seus sintomas e quanto aos tratamentos por terapias complementares.

A ausência de ações públicas voltadas para esta população, o baixo preparo e desconhecimento dos enfermeiros à respeito do uso de terapias complementares, colabora para 
Research, Society and Development, v. 9, n. 10, e8179109213, 2020

(CC BY 4.0) | ISSN 2525-3409 | DOI: http://dx.doi.org/10.33448/rsd-v9i10.9213

que essas usuárias venham a utilizar de outros meios de tratamentos com farmacêuticos, trazendo diversos efeitos colaterais, ou até mesmo tratamentos não convencionais que possam vir a agravar os sintomas do climatério.

Dessa forma, o estudo aponta a necessidade de políticas públicas voltada para esse grupo especifico. Espera-se que essa pesquisa chame atenção para a necessidade de capacitação adequada dos profissionais na área da saúde, principalmente o enfermeiro, que lida diretamente com este grupo, para assim buscarem estratégias para trabalhar e aplicar essas terapias complementares, visando um tratamento mais seguro e saudável para as usuárias.

Em suma, a revisão evidenciou diversos pontos de alta relevância para possíveis pesquisas e trabalhos científicos, como um aprofundamento do funcionamento das terapias complementares em combate à diversas doenças e sintomas do corpo humano; Os impactos da falta de informação à respeito da saúde da mulher e quais as formas de resolver esse problema; Verificação do real impacto da falta de conhecimento dos enfermeiros à respeito de tratamentos alternativos visando a redução da lotação hospitalar. Observa-se que diversos temas podem ser adaptados e desenvolvidos a respeito da saúde da mulher e a desinformação no Brasil.

\section{Referências}

Barra A. A., Albergaria D. A., Mariano F. M. Dantas J. B. Pinto K. M. C. Resende, N. M. (2014). Terapias alternativas no climatério. Janeiro/fevereiro 2014, 42(1), Revista Femina. ID: lil-749138

Brasil. Ministério da Saúde. (2018). Manual de implantação de serviços de práticas integrativas e complementares no SUS / Ministério da Saúde. Secretaria de Atenção à Saúde. - Brasília: Ministério da Saúde. 8-9. Recuperado de: http://189.28.128.100/dab/d ocs/portaldab/publicacoes/manual_implantacao_servicos_pics.pdf

Brasil. Ministério da Saúde. (2015). Política Nacional de Práticas Integrativas e Complementares no SUS: atitude de ampliação e acesso / Ministério da Saúde. Secretaria de Atenção à Saúde. Departamento de Atenção Básica. (2a ed.), Brasília: Ministério da Saúde. p .9.Recuperado de :http://bvsms.saude.gov.br/bvs/publicacoes/politica_nacional_praticas_integ rativas_complementares_2ed.pdf. 
Brasil. Ministério da Saúde. (2016). Protocolos da Atenção Básica: Saúde das Mulheres / Ministério da Saúde, Instituto Sírio-Libanês de Ensino e Pesquisa - Brasília: Ministério da Saúde. p. 230. Recuperado de: https://dab.saude.gov.br/portaldab/biblioteca.php? conteudo=publicações/protocolos_ab).

Curta, Julia, Costa. Weissheimer, \& Anne, Maria. (2020). Percepções e sentimentos sobre as alterações corporais de mulheres climatéricas. Revista Gaúcha de Enfermagem. DOI:10.1590/1983-1447.2020.20190198

Ercole, F. F., Melo, S. L., \& Alcofarado, C. G. L. C. (2015). Integrativereview versus systematicreview. Reme: Revista Mineira de Enfermagem. [s.1], 8(1), 1-3. GN1 Genesis Network. DOI:http://dx.doi.org /10.5935/1415-2762.20140001.

Gelatti, G. T., Oliveira, K. R., \& Colet, C. F. (2016). Potenciais interações relacionadas ao uso de medicamentos, plantas medicinais e fitoterápicos em mulheres no período do climatério. J. res. fundam. care. online. abr.jun. DOI: 10.9789/2175-5361. 2016.v8i2.4328-4346.

Girão, Á. C., Alves, M. D. S., Alves, \& Souza, Â. M., et al. (2014). Acupuntura no tratamento da ansiedade no climatério: terapêutica complementar na promoção da saúde mental. Rev enferm UFPE on line., Recife, 8(6),1538-44, jun. DOI:10.5205/reuol.5876-50610-1SM.0806201412

Leão, R. E., Silva, M. J. P., Salles, F. L., Geaponese, A. L. L., \& Kurebayashi, L. F. S. (2015). Terapias complementares na redução de sintomas do climatério: Ensaio clínico. Cad. de Naturol. Terap Compleme. 4(6). DOI:10.19177/cntc.v4e6201511-19

Moreira, M. A., \& Braitt, L. L. (2014). As alterações biopsicossociais no climatério e a interrelação com a qualidade de vida: um estudo de revisão integrativa. Rev Memorialidades, 21, 57-83. [57] DOI:10.21675/2357-707X.2020.v11.n1.2450

Oliveira, Z. M., Vargens, O. M. C., \& Acioli, S., et al. (2017). Cuidado de enfermagem no climatério: perspectiva desmedicalizada a na atenção primária de saúde. Rev enferm UFPE online., Recife, 11(Supl. 2):1032-43, fev., 2017. DOI: 10.5205/reuol.10263-91568-1RV.1102sup201720. 
Piecha, V. H., Ebling, S. B. D., \& Pieszak, G. M., et al. (2018). Percepções de mulheres acerca do climatério. Rev. Pesqui. Cuid. Fundam. (Online), v.10, 2018. DOI:10.9789/2175-5361. 2018.v10i4.906-912

Poluzzi, E., Piccinni, C., Raschi, E., Rampa, A., Recanatini, M., \& De Ponti, F. (2014). Phytoestrogens in postmenopause: the state of the art from a chemical, pharmacological and regulatory perspective. Current medicinal chemistry, 21(4), 417-436. Doi: 10.2174 / 09298673113206660297.

Rocha, B., Pereira, M. do S., \& Carneiro, J. (2018). Terapias complementares: fitoterapia como opção terapêutica no climatério e menopausa. Revista De Ciências Da Saúde Nova Esperança, 16(1), 16-25. DOI:10.17695/issn.2317-7160.v16n1a2018p16-25

Shifren, J. L., \& Gass, M. L. (2014). The North American Menopause Society recommendations for clinical care of midlife women. Menopause, 21(10), 1038-1062. Doi: 10.1097/gme.0000000000000319.

Silva Araujo, J. B. da, Santos, G. C. dos, Nascimento, M. A., Grippo Dantas, J. da S., \& Carneiro Ribeiro, A. S. (2015). avaliação da intensidade da sintomatologia do climatério em mulheres: inquérito populacional na cidade de Maceió, alagoas. Caderno De Graduação Ciências Biológicas E Da Saúde - UNIT - ALAGOAS,2(3),101-111. Recuperado de https://periodicos.set.edu.br/fitsbiosaude/article/view/2092.

Silva, C. B., Busnello, G. F., Adamy, E. K., \& Zanotelli, S. S. (2015). Atuação de enfermeiros na atenção às mulheres no climatério. Revista Enfermagem UFPE Online, 312-8 DOI: 10.5205/reuol.5221-43270-1-RV.0901 supl201508

Silva, S. B., Nery, I. S., \& Carvalho, A. M. C. (2016). Representações sociais elaboradas por enfermeiras acerca da assistência à mulher climatérica na atenção primária. Revista Rede de Enfermagem do Nordeste, 363-71 DOI: 10.15253/2175-6783.2016000300009. 
Silva, V. H., Rocha, J. S. B., \& Caldeira, A. P. (2016). Fatores associados à autopercepção negativa de saúde em mulheres climatéricas. Revista Ciência \& Saúde Coletiva. DOI: $10.1590 / 1413-81232018235.17112016$

Souza, A. S., Junior, O. C. R., Ribeiro, J. S. S., Mendonça, L. B., Melo, J. M. R., \& Araújo, T. S. (2020). A utilização de fitoterápicos no manejo de mulheres no climatério/menopausa. Research, Society and Development. DOI:10.33448/rsd-v9i9.7416

Souza, S. S., Santos, R. L., Santos, A. D. F., Barbosa, M. O., Lemos, I. C. S., \& Machado, M. F. A. S. (2017). Mulheres e climatério: concepções de usuárias de uma unidade básicas de saúde. Sociedade Brasileira Reprodução Humana. DOI:10.1016/J.RECLI.2017.01.001

Vieira, T. M. M., Araujo, C. R., Souza, E. C. S., Costa, M. A. R., Teston, E. F., Benedetti, G. M. S., \& Marquete, V. F. (2018). Vivenciando o climatério percepção e vivencias de mulheres atendidas na atenção básica. Revista Enfermagem em Foco. DOI:10.21675/2357707X.2018.v9.n2.1084

\section{Porcentagem de contribuição de cada autor no manuscrito}

Thaíse Maria Isnaider Vieira Pilar - 33,3\%

Vanessa Bonfim Mendes - 33,3\%

Nancy Nay Leite de Araújo Loiola Batista - 33,3\% 